\title{
Emergence of spatial transitions in urban congestion dynamics
}

\author{
Aniello Lampo ${ }^{1,2^{*}}$ (D) Javier Borge-Holthoefer ${ }^{1}$, Sergio Gómez ${ }^{2}$ and Albert Solé-Ribalta ${ }^{1,3}$
}

\section{${ }^{*}$ Correspondence:}

nello.lampo@gmail.com

${ }^{2}$ Departament d'Enginyeria

Informàtica i Matemàtiques,

Universitat Rovira i Virgili,

Tarragona, Catalonia, Spain

Full list of author information

is available at the end of the

article

\begin{abstract}
The quantitative study of traffic dynamics is crucial to ensure the efficiency of urban transportation networks. The current work investigates the spatial properties of congestion, that is, we aim to characterize the city areas where traffic bottlenecks occur. The analysis of a large amount of real road networks in previous works showed that congestion points experience spatial abrupt transitions, namely they shift away from the city center as larger urban areas are incorporated. The fundamental ingredient behind this effect is the entanglement of central and arterial roads, embedded in separated geographical regions. In this paper we extend the analysis of the conditions yielding abrupt transitions of congestion location. First, we look into the more realistic situation in which arterial and central roads, rather than lying on sharply separated regions, present spatial overlap. It results that this affects the position of bottlenecks and introduces new possible congestion areas. Secondly, we pay particular attention to the role played by the edge distribution, proving that it allows to smooth the transitions profile, and so to control the congestion displacement. Finally, we show that the aforementioned phenomenology may be recovered also as a consequence of a discontinuity in the node's density, in a domain with uniform connectivity. Our results provide useful insights for the design and optimization of urban road networks, and the management of the daily traffic.
\end{abstract}

Keywords: Urban systems, Road networks, Congestion, Phase transitions

\section{Introduction}

The sustainability of urban life represents one of the greatest challenges of our time. This has sparked a lot of interest among researchers coming from several areas, and has led to the birth of the so-called science of cities (Batty 2012). In this context, the engineering and optimization of road transportation networks stands as a crucial task to ensure the efficiency of traffic dynamics and to control the related congestion emergence.

In the literature, the study of road transportation networks has been addressed following different methods, according to the purpose and the system characteristics. For instance, the phenomenology emerging on arterial roads (also named inter-urban, peripheral or high-capacity roads), characterized by long segments and limited interconnections, has been treated by means of fluid models (Helbing 2015), or the fundamental diagram of traffic flow (Godfrey 1969; Daganzo and Geroliminis 2008). The author(s) and the source, provide a link to the Creative Commons licence, and indicate if changes were made. The images or other third party material in this article are included in the article's Creative Commons licence, unless indicated otherwise in a credit line to the material. If material is not included in the article's Creative Commons licence and your intended use is not permitted by statutory regulation or exceeds the permitted use, you will need to obtain permission directly from the copyright holder. To view a copy of this licence, visit http:// creativecommons.org/licenses/by/4.0/. 
case of central streets (also called intra-urban), instead, has been traditionally analyzed by means of network science (Guimerà et al. 2002; Solé-Ribalta et al. 2018; Chen et al. 2020).

These two types of road networks, which are usually studied independently, are increasingly intertwined as cities sprawl over suburban areas. Therefore, an entanglement perspective is of great importance to properly deal with the dynamics of vehicles flux, as well as other related phenomena. In Lampo et al. (2021), we pursued this path and focused on the spatial behavior of congestion onset. We found that the structural discontinuity between the central and arterial roads yields an abrupt transition of the location where congestion occurs. In particular, congestion points shift away from the city center as larger areas are included in the urban spatial domain. Remarkably, such property is also detected in almost one hundred real cities road networks worldwide.

This result represents an important resource to improve the efficiency of road networks. The displacement of the traffic bottlenecks towards the peripheral zones, indeed, is crucial to reduce the pressure on the city center, and to avoid the degradation of the transportation system. Therefore, the possibility to control and manipulate such effect constitutes a notable task which has never been performed before. The current paper goes in this direction. Specifically, we present a series of extensions of the theoretical setup in Lampo et al. (2021), aimed to approach more realistic situations, and shed light on the fundamental ingredients ruling the observed phenomenon.

In Lampo et al. (2021), the spatial separation between urban center and the surrounding periphery was recognized to be a necessary requirement for the emergence of congestion transitions. There, the road transportation networks are treated as the inextricable blending of two different structures, that are however sharply separated in space. Here, instead, we look into the consequences of a spatial overlap between center and periphery, namely we introduce a hybrid region where the dense and highly regular frame of central roads coexists with that of the arterial ones. This is a crucial point to match real contexts, since the transition between center and periphery may not be sharp, but it is expected to occur in a wider spatial range.

Along this line, we investigate the role of the edge distribution, implementing the most general case of a non-uniform spatial-dependent density. This turns to be a key object to manipulate the location of congestion onset, allowing to smooth its shift from the center. The edge distribution, i.e., the number of edges over the unit area, may also be manipulated by modifying the number of nodes. Thus, we pay great attention to the situation in which connectivity is assumed to be uniform, while urban center and periphery are constructed as regions with a high and low concentration of nodes, respectively. In this way, we recover the abrupt transitions of congestion, and highlight the importance of both node and edge distributions to the control of traffic bottlenecks.

The manuscript is organized as follows. In "A model for center-periphery entanglement" section we present the DT-MST random planar model developed in Lampo et al. (2021), constituting a practical benchmark to deal with the interplay of central and arterial roads. The former are described using a Delaunay triangulation (DT), while the latter are treated using a minimum spanning tree (MST), both embedded on separated spatial domains. In "Spatial abrupt transitions of congestion" section we employ this model to study the behavior of the congestion radius as a function of the urban size, showing 


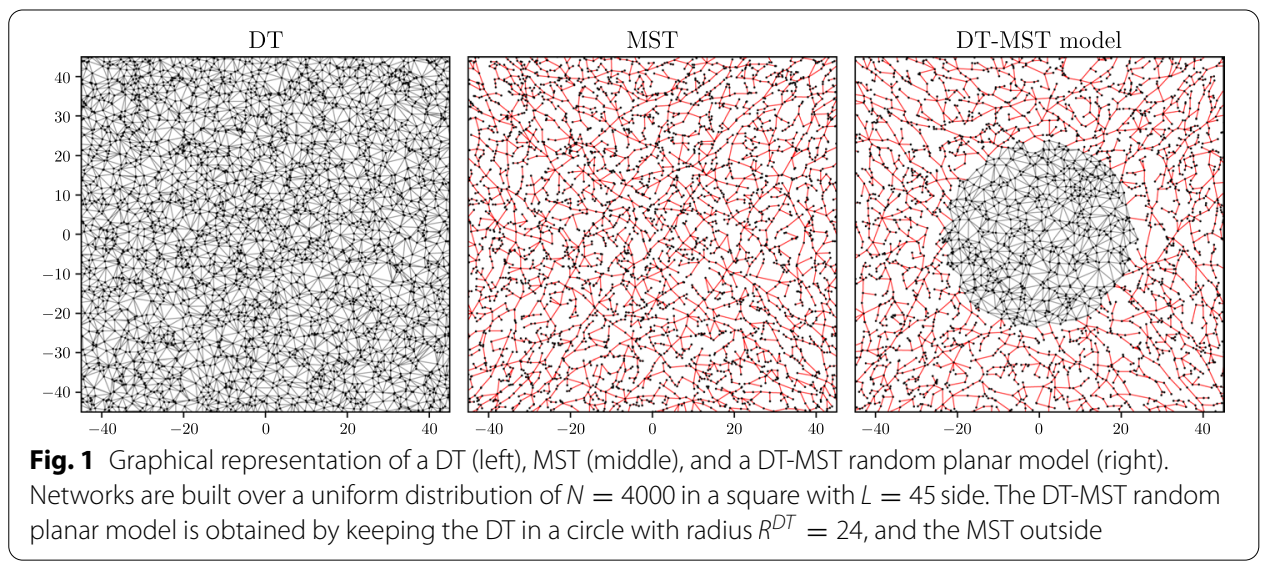

that it is possible to detect two regimes, which correspond to bottlenecks located in the center, and in its connection with the periphery, separated by an abrupt transition. In "Center-periphery entanglement with spatial overlap" section we consider the more realistic situation where MST and a DT are not sharply separated and can share a finite portion of space. We find that this yields a second transition, as a consequence of the further structural discontinuity introduced by the new hybrid region. In "Center-periphery entanglement with non-uniform edges distribution" section we extend the previous cases by including the possibility of a non-uniform edge distribution, and show that it leads to smoother transitions. Finally, in "Center-periphery entanglement with non-uniform nodes distribution" section we investigate the inverse situation in which center and periphery emerge as areas with different node densities, but maintain the same connectivity. This situation enables the recovery of the abrupt transition and highlight the role of nodes distribution. The importance of these results for urban planning, as well as possible future extensions, are discussed in "Conclusions and perspectives" section.

\section{A model for center-periphery entanglement}

Road transportation networks consist of networks whose edges represent roads, while the points where they intersect constitute the nodes. Road networks are a paradigmatic example of planar graphs, namely graphs which can be embedded on a surface in such a way that no edges cross each other; of course, real road networks may have crossing edges, but they always represent a very small fraction of the whole system. In this section we present a general class of planar graphs that, while being as free as possible of any microscopic constraints, keeps the distinctive feature of an entanglement between central and arterial roads.

The most natural choice to model urban center, characterized by a dense web of streets resembling short segments, is provided by a Delaunay triangulation (DT). For a given set of points in a plane, DT is a triangulation over the plane, i.e., any triad of neighboring points defines a triangle, such that no point lies within the circumcircle of any triangle. An example of DT is depicted in Fig. 1 for a random uniform distribution of points. Roughly speaking, DT yields a network structure characterized by a high number of shorts edges, uniformly spread over all the nodes (regularity), with a great redundancy of paths. Remarkably, there are no edge overlapping, i.e., DT preserves planarity. 
Similar properties could be recovered by considering other types of regular graphs, such as grids, but these would impose a spatial constraint on the nodes positions, making the model contrived. However, as widely discussed in Lampo et al. (2021), this kind of structure produces equivalents results for congestions.

Peripheral arterial roads are high-capacity streets whose primary function is to deliver traffic between different urban centers. As such, intersections are often reduced to improve traffic flow, and the resulting peripheral street frame resemble a sparse web of long segments. Still, we seek for a network structure where these properties emerge automatically. The low connectivity suggests to look into a tree structure, whose general implementation is provided by a minimum spanning tree (MST).

The MST is defined as the subset of the edges (of a connected, edge-weighted undirected graph) connecting the vertices in such a way that there are no redundant paths, and minimizing the total edge weight. In our model, the construction of the MST is obtained from a DT, in which the edge weights are the reciprocals of their corresponding edge-betweenness, rather than the Euclidean distance between their adjacent nodes. This is a crucial aspect to reproduce the main routing backbones of arterial periphery webs, as shown in Fig. 1 (middle). A distance-based MST, like that employed in Kirkley et al. (2018), does not consider routing flows, which are essential in transportation networks.

The model we propose to treat central and arterial peripheral roads in a unified entangled framework is constituted by a DT core surrounded by a MST. Summarizing, the resulting synthetic network is built according to the following steps:

- We consider a uniform random distribution of $N$ points (the nodes) in the spatial domain $\mathcal{I}=[L,-L] \times[L,-L]$

- This set of points induces a DT over $\mathcal{I}$. We hold it within the circle of radius $R^{(D T)}$, i.e., for points with radius $r \leq R^{(D T)}$;

- Out of such a circle, for $r>R^{(D T)}$, we look for the MST resulting from the DT, obtained by minimizing with respect to the reciprocals of the edge betweenness of the links.

Our model, coined as DT-MST random planar model, is so defined by only three parameters: the square spatial side length $L$; the number of nodes $N$; and the DT radius, $R^{D T}$. In Fig. 1 (right) we present a realization of the model for a given choice of these parameters.

The idea to model central and peripheral roads as a DT and a MST, respectively, may be better understood in terms of betweenness distribution. This has been carefully studied in Kirkley et al. (2018) for a significant number of cities worldwide, proving that it exhibits an invariant bimodal structure. Such bimodal character is a first indicator of the existence of a structural discontinuity between different topologies as we depart from the city center. Specifically, as discussed in Sect. 3 of Lampo et al. (2021), the two branches of the betweenness distribution may be fitted with those of a tree and a DT respectively, providing an endorsement of the DT-MST model. 


\section{Spatial abrupt transitions of congestion}

We study first the behavior of congestion in the center-periphery intertwined framework introduced above. The quantitative assessment of congestion may be performed by recalling betweenness centrality. There exists a large set of studies about traffic (Guimerà et al. 2002; Zhao et al. 2005; Echenique et al. 2005; Yan et al. 2006; Liu et al. 2007; Dong et al. 2012; Tan et al. 2014; Manfredi et al. 2018) where the critical injection rate of vehicles $\gamma_{c}$, i.e., the maximum rate at which vehicles can enter the system without congesting it, can be evaluated as

$$
\gamma_{c}=\frac{N-1}{B_{n}^{*}} .
$$

where $N$ is the nodes number of the street network and $B_{n}^{*}$ represents the maximum node betweenness.

The analysis of congestion in terms of betweenness permits to evaluate how congestion is affected by the underlying network structure. It is worth highlighting that the definition of betweenness embodies a routing protocol which implicitly assumes a traffic dynamic, relying on shortest-paths, and an Origin-Destination matrix which in our case is assumed to be uniform and all-to-all. Thus, combining these two ingredients the traffic model can be defined with precision. Previous works (Solé-Ribalta et al. 2018, 2016a, b, 2019) show the exact correspondence between the betweenness centrality as a measure to estimate node and edge traffic load, and the results obtained through the use of agent-based models (the actual traffic model).

Equation (1) suggests that the maximum betweenness node marks the congestion onset. We are interested in its geographic location, that can be quantified by the distance to the center (radius) of the maximum betweenness node, hereafter called the congestion radius, $R_{c}$.

We start by exploring the dependence of the congestion radius $R_{c}$ on the patch radius $R_{p}$ for the DT and MST networks. This represents our baseline case, where no DT-MST entanglement exists, i.e. the structural organization of cities is spatially uniform. Results are presented in Fig. 2 (right). In both situations, the congestion radius remains flat across $R_{p}$ values, and exhibits a very low value compared with the size of the system $L$. This means that congestion mainly occurs in the city center, regardless of the selected urban area. Such behavior is also reflected in the left panel, where hexagonal bins provide information about the location of maximum betweenness nodes.

It is important to underline that the $R_{c}-R_{p}$ relationship in Fig. 2 is studied over an ensemble of realizations of the DT (top) and MST (bottom) models, in order to obtain a statistically solid result as free as possible of any special configuration. The final value of the $R_{c}$ quantity, shown in Fig. 2, is extracted by looking into the statistical mode over the realization set. Shadow areas portray the mean square displacement from the statistical mode considering all the realizations. To calculate the statistical mode, since $R_{c}$ takes continuous values, we first aggregate the values into bins and the presented results are with respect to those bins. A very similar plot is obtained when considering the average rather than the mode, as shown in reference (Lampo et al. 2021).

We now explore how the location of the congestion radius is affected by the intertwined model we present here. To this end, we calculate $R_{c}$ for subgraphs of the DT-MST 

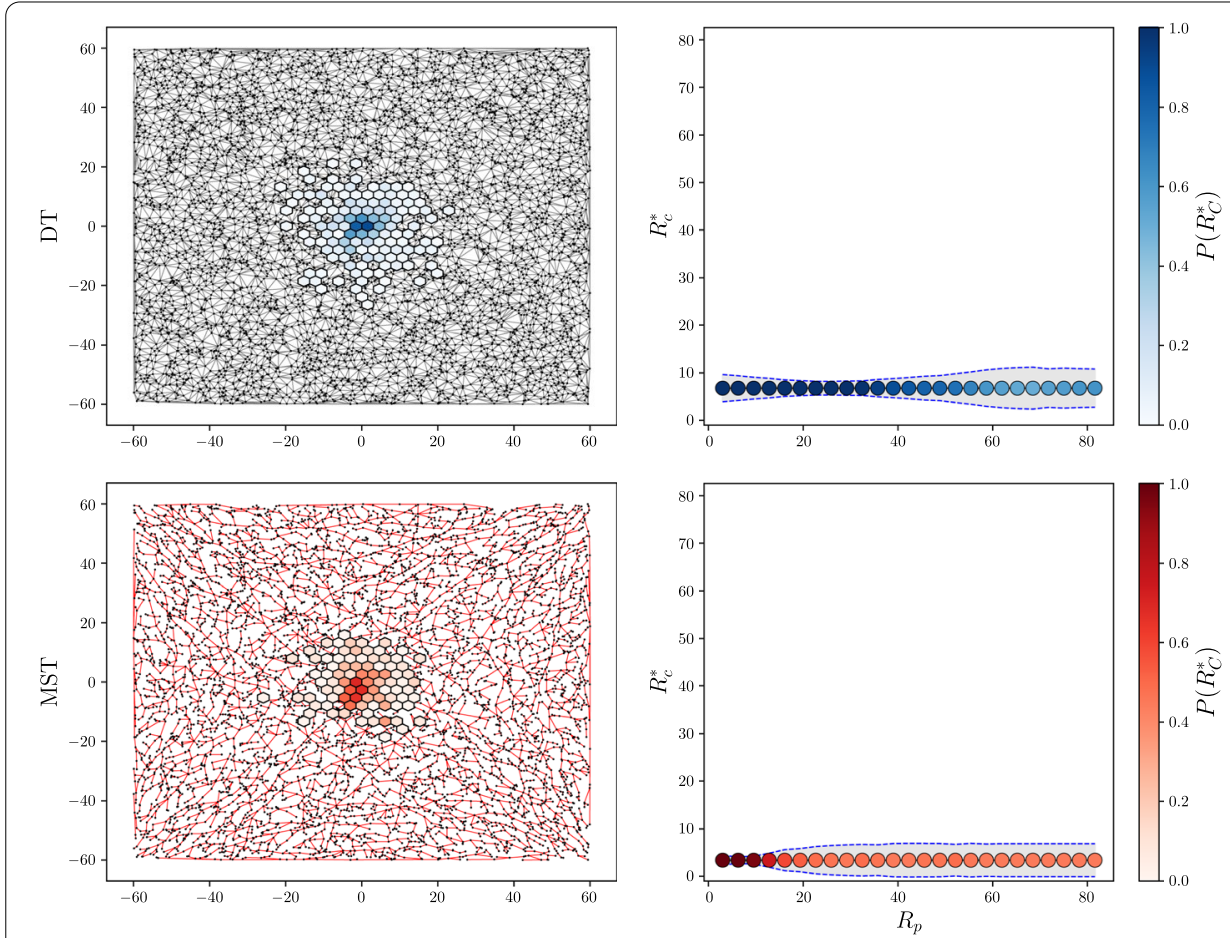

Fig. 2 Spatial behavior of congestion for a DT (top) and a MST (bottom) networks, built on a uniform distribution of $N=6000$ nodes in a square with side length $L=60$. Left column contains the graphic representations of the networks. Hexagonal bins provide information about the occurrence of congestion nodes in space, and their colors show the related frequency in proportion to the intensity. In the right column we present the dependence of the congestion radius $R_{c}$ on the patch radial size $R_{p}$, i.e., the radius of the circle centered in the middle of the network, that is used to define the considered subgraph within this radius. The congestion radius range is divided in bins, and each point is located at the statistical mode $R_{c}^{*}$ obtained with the distribution of $R_{c}$ after 100 realizations. The color of the circular markers shows the probability of that value over the experimental $R_{c}$ distribution. Shadow areas represent the variance of the different realizations of $R_{c}$ values with respect to the bin average value. Importantly, congestion mostly occurs in the center and no transitions arise, suggesting that these are a consequence of the intertwining of different graph structures

contained in concentric circles with radius $R_{p}$. Low values for this patch radius $R_{p}$, e.g., $R_{p}<R^{(D T)}$, refer to situations where the arterial periphery is suppressed, while large values, i.e., $R^{(D T)}<R_{p} \lesssim L$, describe an urban context where arterial periphery is relevant.

Results are shown in Fig. 3, where we repeat the same experiment but for the DT-MST random planar model. The relationship between congestion radius and patch one is not constant anymore, but depicts a step pattern configuration, namely an abrupt transition between two regimes associated to congestion at the DT center and at its connection with the MST. The former emerges for small patches size, while the latter arises at large ones, i.e., when the arterial roads contribute significantly to the network traffic. It is important to remark that, in addition to the structural discontinuity between center and periphery, a significant peripheral flux is required. That is, a proper balance between the size (in number of nodes) of the Delaunay center and the arboreal periphery. See for instance the particular case of Fig. 3, where $R^{(D T)}=24$. If the patch radius is less than or equal to such a value, namely $R_{p} \leq R^{(D T)}=24$, we are only considering the Delaunay center and the results reduces to the experiments in Fig. 2. The situation 

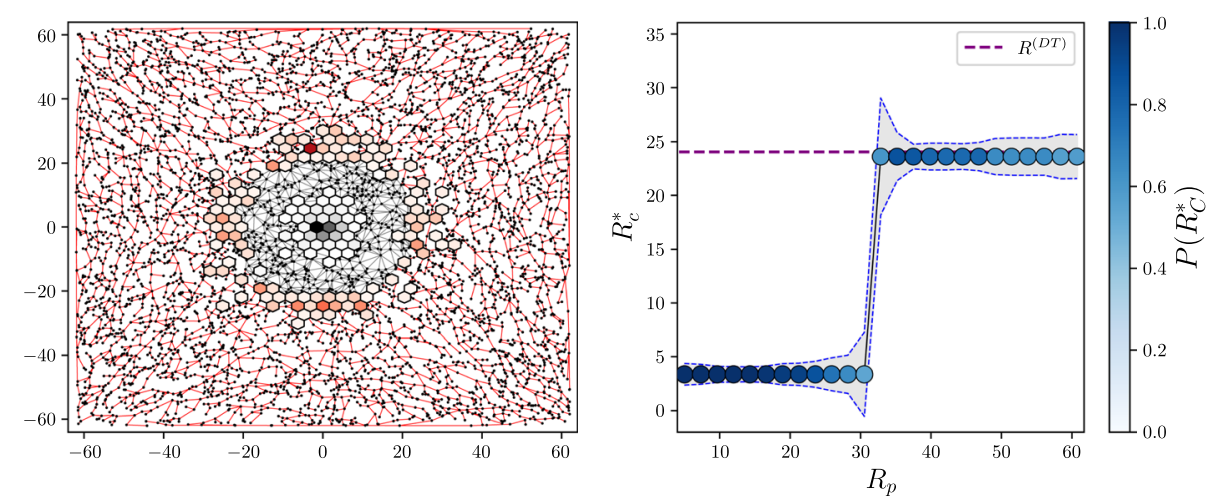

Fig. 3 Spatial behavior of congestion nodes for the random planar models introduced in "A model for center-periphery entanglement" section with $N=6000, L=60$, and $R^{(D T)}=24$. In the left panel, the resulting network configuration, with MST and DT edges painted in red and black, respectively. Hexagonal bins provide information about the occurrence of congestion nodes in space, and their colors show the related frequency in proportion to the intensity. In the right panel, we present the dependence of the congestion radius $R_{c}$ on the patch radial size $R_{p}$, i.e., the radius of the circle centered in the middle of the network, that is used to define the considered subgraph within this radius. The congestion radius range is divided in bins, and each point is located at the statistical mode $R_{c}^{*}$ obtained with the distribution of $R_{c}$ after 100 realizations. The color of the circular markers shows the probability of that value over the experimental $R_{c}$ distribution. Shadow areas represent the variance of the different realizations of $R_{c}$ values with respect to the bin average value

in which $R_{p}$ gets larger than $R^{(D T)}$, while still keeping a very close value, is still similar, because the network practically reduces to a DT core with a few surrounding nodes, which slightly increment the betweenness of the DT frontier nodes. These small increments become irrelevant for the determination of the onset of congestion nodes (those with largest betweenness). When $R_{p}$ is large, and the MST acquires a significant size, we finally detect the transition. Figure 3 permits to visually infer the critical MST size necessary for the emergence of transition (for the given model realization): $R_{p}$ approximately equal to 30 .

The comparison of this results with Fig. 2 suggests that the abrupt transition is due to the interplay of the composing structures, and in particular to the spatial discontinuity produced in the network. Note that one could also implement the DT-MST entanglement by homogeneously overlapping the edges of the former an arboreal skeleton, without any spatial separation, and, as discussed in Lampo et al. (2021, Fig. S10), no transition is detected. This is a crucial aspect that will be explored in the rest of the paper.

We finally would like to remark that the analysis of the dependence of $R_{p}$ with respect to the congestion radius may mimic two important urban process, as already discussed in detail in Lampo et al. (2021). In many situations, especially those of European cities with a strong historical background, urban settings seem to evolve from a dense center, exhibiting high regularities, towards a sparse, tree-like structure (Masucci et al. 2013). In the framework of our model, we can emulate this type of urban sprawl by fixing the Delaunay and MST size, and studying their mutual proportion, namely by tuning the patch radius $R_{p}$. In this context, it is worth highlighting as well the work by Strano et al. (2009), where a similar urban growth process is 
discussed, with the ratio between the size of center and the periphery also plays an important role.

At a much faster timescale, we can find a relationship between our analysis and daily traffic patterns. During the morning early rush hours, citizens depart from dormitory cities towards the urban center. These dormitory cities are usually located in the periphery, thus the roads employed to reach the city center are mostly peripheral. This may be reproduced in our framework by a network where the spatial extension of the arboreal periphery is much larger than that of the Delaunay center. In the later stages of the rush hours, less traffic is observed in the outer parts of the city, concurrent to an increase in the urban ones: citizens are close to reach their destinations in the center. This can be modeled by reducing the proportion between periphery and center. Similar to the urban sprawl analysis, the same effect may be produced by tuning the patch radius. Small values for the patch radius will consider a minimal fraction of the MST, while large ones will consider a predominant MST flux.

\section{Center-periphery entanglement with spatial overlap}

The spatial discontinuity between the two topologies, resulting from their embedding on separated regions, is a fundamental ingredient to obtain the abrupt transition. So far we have considered a sharp separation between DT and MST. However, this is an extremely idealized situation, since the transition between a dense center to a sparse periphery usually occurs in a wider spatial range.

In this section we maintain the general separation between DT and MST but include the possibility of a spatial overlap in a finite interval. This is obtained by introducing a hybrid region with intermediate connectivity properties. The new region is ring-shaped, with small and large radius respectively given by $R^{(D T)}$ and $R^{(D T)}+\Delta R$, where $\Delta R>0$ denotes the width of the ring. In this region, we cover the MST skeleton with a density $\pi \in[0,1]$ of DT edges, sampled from a uniform distribution. The extreme bound $\pi=0$ refers to the situation in which no edge has been added, while $\pi=1$ corresponds to a completely saturated region.

This class of networks is shown in the left column of Fig. 4, for different values of the parameter $\pi$. The corresponding relationship between the congestion radius and the patch one is presented in the right column. The cases with extreme values $\pi=0$ and $\pi=1$ fall into the category treated in the previous section, with DT radius equal to $R^{(D T)}$ and $R^{(D T)}+\Delta R$, respectively. These exhibit one abrupt transition between two regimes related to congestion in the center and in its frontier with the periphery.

Interesting phenomenology arises for intermediate values of $\pi$. In these cases, the hybrid region is not saturated and differs from both the DT and MST. This induces a further structural discontinuity in the network, which affects the spatial behavior of congestion. Particularly, a second abrupt transition stands out: as $R_{p}$ increases, congestion points abruptly shift from the center to the DT frontier $\left(R_{c} \approx R^{(D T)}\right)$, and then to that of the hybrid ring $\left(R_{c} \approx R^{(D T)}+\Delta R\right)$. The spatial overlap, thus, induces a further possible congestion location.

This experiment sheds light on the mechanism producing the abrupt transition. The structural discontinuity required to obtain such a phenomenon consists of a sharp 

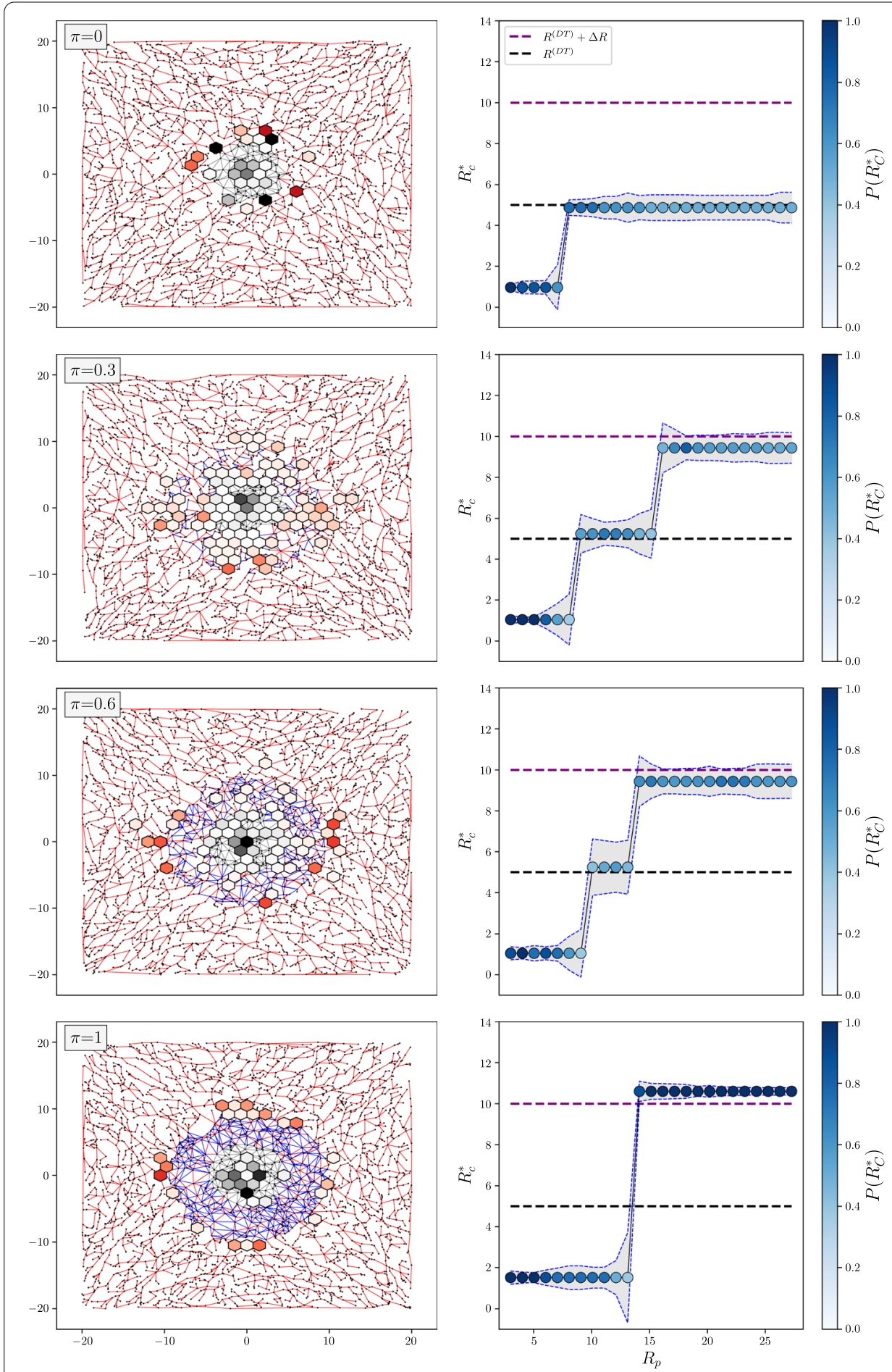

Fig. 4 Spatial behavior of the congestion nodes for the setup introduced in "Center-periphery entanglement with spatial overlap" section. The figure is organized similarly to Figs. 2 and 3: left panels show the network configurations, where blue edges refer to the $\pi$ density of further DT links we add on top of the MST skeleton in the $[R, R+\Delta R]$ hybrid region. Still, hexagonal bins describe the occurrence of congestion in space. Right panels presents the dependence of congestion radius on the patch one. Here, circles are located at the statistical mode over an ensemble of 100 realizations of different random distributions of $N=3000$ nodes in a square of side equal to $L=20$, with $R^{(D T)}=5$, and $\Delta R=5$. The figure highlights the emergence of a further intermediate congestion regime, as a consequence of the hybrid region marked in blue 
change of the spatial density of edges, i.e., number of edges over the unit area. Then, one may suppose that such quantity permits to manipulate the abruptness of the congestion transition; this is the aim of "Center-periphery entanglement with nonuniform edges distribution" section. Additionally, the spatial edge density can also be modified by acting on the nodes distribution, rather than network connectivity; this will be explored in "Center-periphery entanglement with non-uniform nodes distribution" section.

The intermediate congestion regime emerged in Fig. 4 portrays a similar pattern to that detected in Lampo et al. (2021), although in a different framework: GT-model. This consists of square grid, representing the city center, connected to four regular trees, one at each side, representing the urban periphery. In this context, three congestion regimes arise, localized respectively in the grid center, grid side and the tree root. This situation is equivalent to that discussed in the current section. the GT-model, indeed, defines three regions with different edges density: the areas where are embedded the grid, the trees and the grid-tree connections. The discontinuity between these regions lead to a multiple congestion pattern.

\section{Center-periphery entanglement with non-uniform edges distribution}

In the previous section we recognized the relationship between the observed phenomenology and the connectivity properties of a road network. Particularly, abrupt transitions follow from sharp discontinuities in the edge density. This suggests that bottlenecks location may be controlled by properly tuning such quantity. This is the purpose of the current section.

So far, the spatial discontinuity in the edge density emerged spontaneously as a consequence of the intertwining of different topologies. However, within a given region the connectivity has been considered constant in space. Particularly, the hybrid region introduced in "Center-periphery entanglement with spatial overlap" section was constructed by covering the MST skeleton with DT edges sampled from a uniform spatial distribution. This constitutes a strong idealization because road density is in general not uniform over the city. Specifically, the transition from a dense center to a sparse arterial periphery depicts a non-trivial profile of the street distribution, interpolating between that of the DT and the MST one.

We propose an extension of the setup in "Center-periphery entanglement with spatial overlap" section, where the DT edges in the hybrid region are sampled from a non-uniform distribution. We assume that an edge with center at radius $r$ has a probability to be added given by

$$
P(r)=\alpha \exp (-k r)+\beta, \quad r \in\left[R^{(D T)}, R^{(D T)}+\Delta R\right],
$$

where $k \neq 0$ is a real constant ruling the damping in space of the exponential. The $\alpha$ and $\beta$ coefficients are fixed by the boundary conditions

$$
P\left(R^{(D T)}\right)=1, \quad P\left(R^{(D T)}+\Delta R\right)=0,
$$

which lead to 

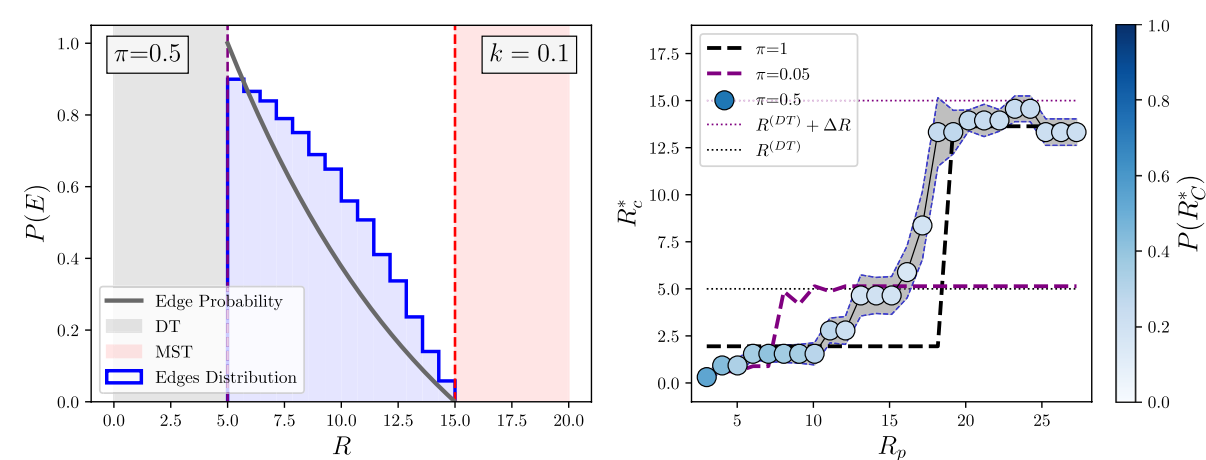

Fig. 5 Congestion radius pattern for the setup introduced in Sec. 5 , with $N=3000, L=20, R^{(D T)}=5$, and $\Delta=10$. Hybrid region is generated by overlapping a fraction $\pi=0.5$ of DT edges sampled from the distribution in Eq. (2), with $k=0.1$. In the left panel, the blue histogram describes the final distribution of these edges. The right panel reports the congestion radius $R_{c}$ as a function of the parch radius $R_{p}$. The black and purple dashed line report the result related to $\pi=1$ and $\pi=0.05$ respectively, where all the edges and approximately no one have been added, and match the situation described in Sec. 4. The dotted line refers to the edge distribution shown in the right panel, where circles are located at the statistical mode over an ensemble of 100 realizations. In this case transition exhibits a smooth, rather than abrupt, profile as a consequence of the non-tight discontinuity in the edge density

$$
\alpha=\frac{1}{e^{-k R^{(D T)}}-e^{-k\left(R^{(D T)}+\Delta R\right)}}, \quad \beta=\frac{1}{2}\left[1-\frac{e^{-k R^{(D T)}}+e^{-k\left(R^{(D T)}+\Delta R\right)}}{e^{-k R^{(D T)}}-e^{-k\left(R^{(D T)}+\Delta R\right)}}\right] .
$$

Additionally, for generalization purposes and consistency with the method in "Centerperiphery entanglement with spatial overlap" section, we ensure that the final network includes a fraction $\pi$ of all the candidate edges.

This procedure introduces a bias in the construction of the hybrid region, whose edge connectivity interpolates now between that of the DT and the MST. In Fig. 5 (left) the grey solid line refers to Eq. (2) for a given choice of the parameter $k$. The blue histogram portrays the final distribution of a fraction $\pi=0.5$ of the DT edges: their density approximately reproduces the profile in Eq. (2) and is not constant in space but decreases exponentially as a function of the radius. Of course, when $\pi=1$ the hybrid region saturates and gets indistinguishable from the DT core, which extends until $r=R^{(D T)}+\Delta R$. In this case the system reduces to the DT-MST model in "A model for center-periphery entanglement" section. In the right panel we present the dependence of the congestion radius $R_{c}$ as a function of the patch size $R_{p}$. Black (purple) dashed lines refer to $\pi=1(\pi=0.05)$, corresponding to a hybrid region in which all the edges (approximately no one) have been added, and reproduces the abrupt transition pattern discussed in "A model for center-periphery entanglement" section. A new behavior arises at $\pi=0.5$ : the transition between different congestion regimes is not abrupt anymore. Congestion radius, approximately equal to zero at small parches, smoothly grows until $r=R^{(D T)}+\Delta R$, as a consequence of the continuous edge density degrowth shown in the left panel.

A similar behavior is detected in Fig. 6, for a different choice of parameters. Both make use of $k=0.1$, and this deserves to be commented. High values for $|k|$ make the function 

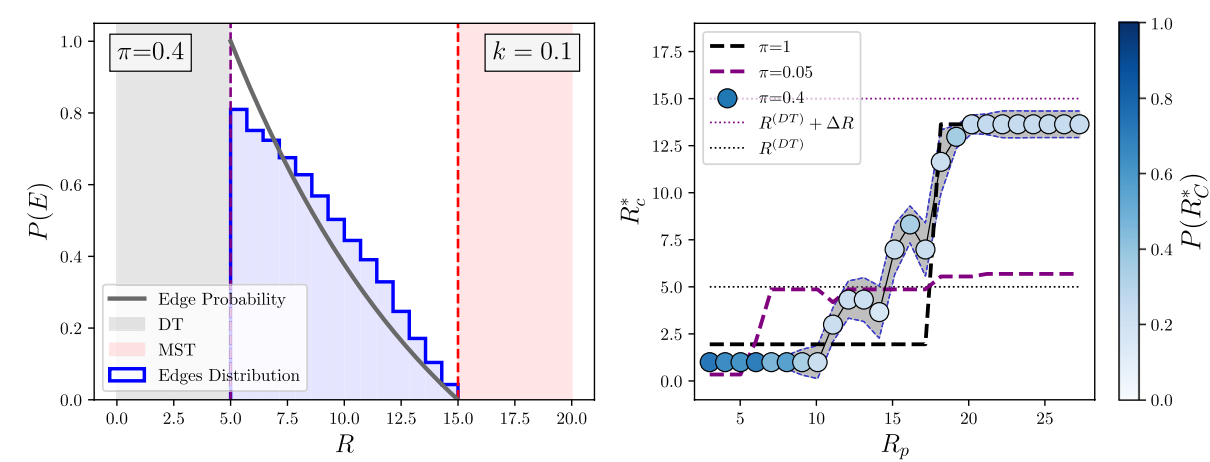

Fig. 6 Congestion radius pattern for the setup introduced in "Center-periphery entanglement with non-uniform edges distribution" section, with $N=6000, L=20, R^{(D T)}=5$, and $\Delta=10$. Hybrid region is generated by overlapping a fraction $\pi=0.4$ of DT edges sampled from the distribution in Eq. (2), with $k=0.1$. In the left panel, the blue histogram describes the final distribution of these edges. The right panel reports the congestion radius $R_{c}$ as a function of the parch radius $R_{p}$. The black and purple dashed line report the result related to $\pi=1$ and $\pi=0.05$ respectively, where all the edges and approximately no one have been added, and match the situation described in "Center-periphery entanglement with spatial overlap" section. The dotted line refers to the edge distribution shown in the right panel, where circles are located at the statistical mode over an ensemble of 100 realizations. In this case transition exhibits a smooth, rather than abrupt, profile as a consequence of the non-tight discontinuity in the edge density

in Eq. (2) elbow-shaped, leading to a quasi-sharp second jump in the edge density, which would reproduce the situation analyzed in "Center-periphery entanglement with spatial overlap" section. In general, the density profile in Eq. (2), with the coefficients in Eq. (4), approaches a constant function with value 1 for $k \rightarrow-\infty$, and with value 0 for $k \rightarrow+\infty$. These two cases fall into the situation described in "Center-periphery entan-

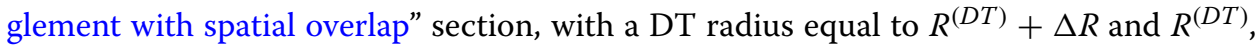
respectively.

\section{Center-periphery entanglement with non-uniform nodes distribution}

The analysis of the previous sections suggests that the existence of multiple congestion regimes follows from different connectivity regions in the road networks. In the most simple case of "A model for center-periphery entanglement" section, this has been implemented by entangling a DT core and a MST periphery, yielding a discontinuity in the edge density and so to different degrees of paths redundancy. We argue that there exist alternative implementations to reproduce this situation.

So far, the network model has been constructed over an uniform distribution of nodes, i.e., a constant nodes density. This leads to considering different topologies in order to produce the wished change in the spatial density of edges. One could also look into the inverse situation, in which center and periphery result from different nodes densities, high in the center and low in the periphery, but exhibiting the same connectivity properties (i.e., the same average degree).

The network we consider in this section has a DT structure, and a different nodes density between center and periphery. The former, defined as a circle with radius $R^{(C)}$, has density $\rho_{c}$, while that of the latter is $\rho_{p}<\rho_{c}$. In the limit in which $\rho_{c}=\rho_{p}$ the network reduces to a uniform DT as that presented in Fig. 2. We assume that both $\rho_{c}$ and $\rho_{p}$ are constant, i.e., they are uniform within their own regions. 

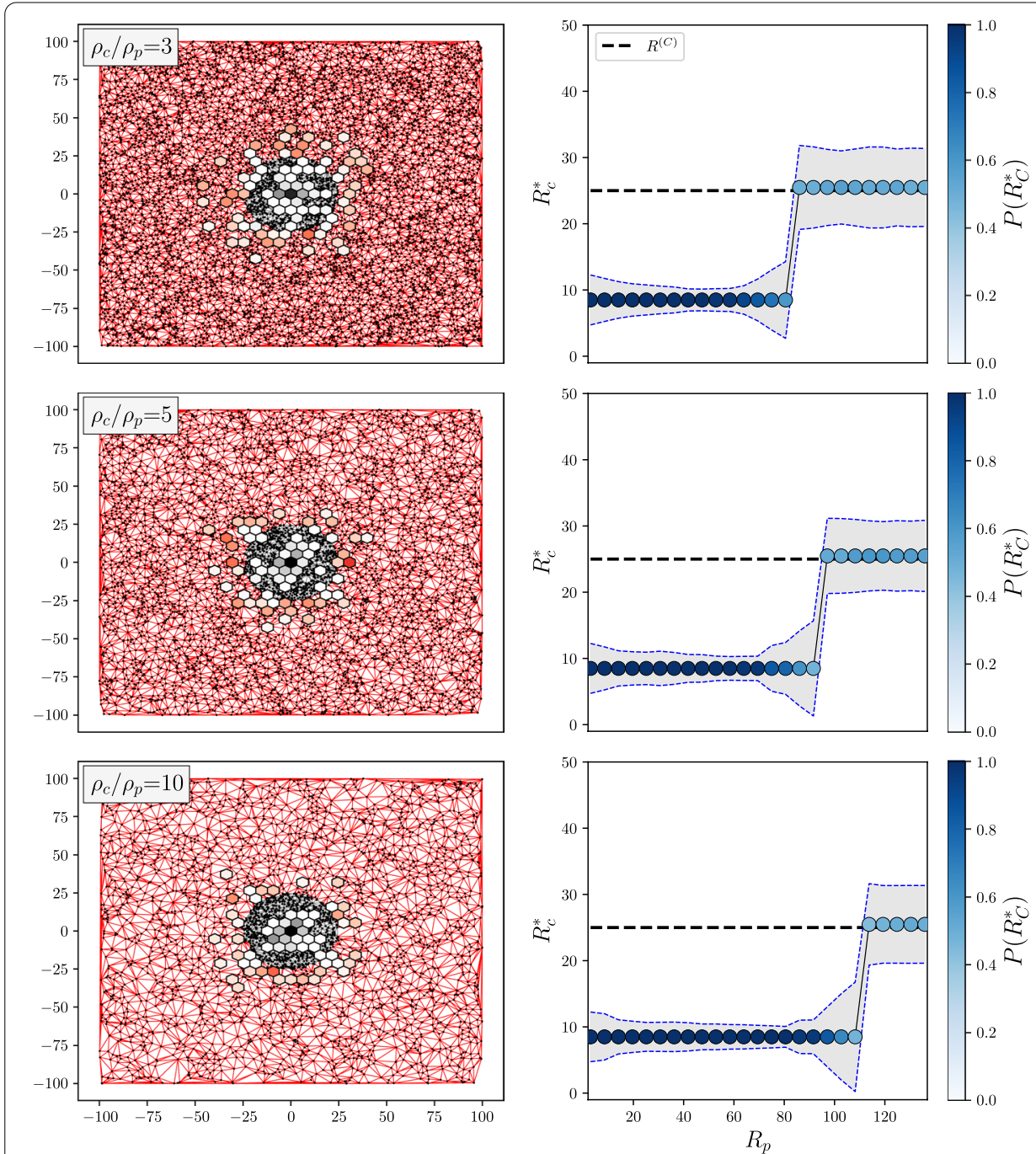

Fig. 7 Spatial behavior of congestions for the network model introduced in "Center-periphery entanglement with non-uniform nodes distribution" section. Left column shows the network configuration, where center and peripheral edges are painted in black and red, respectively. Here, hexagonal bins provide an information about the location of maximum betwenness nodes. Right column presents the dependence of the congestion radius on the patch one. Here, circles are located at the statistical mode over an ensemble of 100 realizations of different random distributions of nodes in a square of side equal to $L=100$, with $R^{(c)}=5$, $\rho_{C}=\frac{N}{\pi\left(R^{(C)}\right)^{2}},(N=10000)$. Rows refer to different density ratio. The figure depicts a clear transition pattern as that detected in Fig. 3

In Fig. 7 we present the spatial behavior of congestion for different center-periphery density ratios $\rho_{c} / \rho_{p}$. It is possible to note a clear abrupt transition between a regime describing congestion in the center, and its connection with the periphery. We remark once more that the periphery is not modeled as a MST, but the whole network results from a DT over the nodes distribution. This confirms the possibility to produce an abrupt displacement of traffic bottlenecks as a consequence of a discontinuity in the nodes density, without any change in the topology.

An important aspect of the behavior in Fig. 7 concerns the pattern at different density ratios $\rho_{c} / \rho_{p}$. The minimum patch radius value allowing to observe congestion in 
the center-periphery connection, grows as the density ratio increases. This is due to the fact that, as already discussed in "A model for center-periphery entanglement" section, the transition requires a large peripheral flux in order to emerge. For a given choice of the system parameters, such flux reduces as the density ratio decreases. We expect, indeed, that when the peripheral number of nodes is low enough, $\rho_{c} / \rho_{p} \rightarrow \infty$, no transition arises, since we approach a network compound by a DT core with a few surrounding points. Therefore, the experiment discussed in this section is constrained to a certain range values of $\rho_{c} / \rho_{p}$ : large enough to produce a structural discontinuity, low enough to ensure a relevant peripheral flux.

\section{Conclusions and perspectives}

In Lampo et al. (2021) we showed that traffic bottlenecks shift away from the city center as larger concentric areas are included in the urban spatial domain, giving rise to abrupt congestion transitions. The current work sheds lights on the main mechanisms which permit to control the phenomena, and provides useful insights in urban planning and the management of traffic.

The analysis in Lampo et al. (2021) points to the structural discontinuity between central and arterial roads as the fundamental ingredient behind the abrupt transition of congestion points. The present paper clarifies the formal meaning of such structural discontinuity and relates it to the spatial density of edges. We show that, acting on this quantity, it is possible to modify the transition pattern, and so to control the geographic occurrence of congestion. Briefly, our results may be summarized as follows. First, the introduction of additional jumps in the edge density is associated to the emergence of new abrupt transitions, ("Center-periphery entanglement with spatial overlap" section). Second, these transitions get smoother if the space-density of DT edges on the MST skeleton exhibits a proper continuous profile ("Center-periphery entanglement with non-uniform edges distribution" section). Third, abrupt transitions may be obtained by means of discontinuities in the nodes density, without any change in the network topology.

These results extend the applicability of the DT-MST model and allows to better interpret the empirical validation presented in Lampo et al. (2021, Sect. 7). There, the congestion radius behavior as a function of the parch size was studied for street networks of 97 cities worldwide. Overall results showed that 52 cities present clear, detectable at naked eye, regimes with abrupt transitions between them. The existence of multiple transitions, rather than a single one, may be understood as the consequence of various edge density discontinuity points in the road networks. Still, 45 cities present detectable regimes with smoother transitions which can be now understood as the results of smooth change in the edge density.

The possibility to control the spatial distribution of the congestion points, and in particular to remove them from the center, constitutes an interesting tool for urban planning. The displacement of the traffic bottlenecks towards peripheral areas is a positive effect to reduce pressure on the center, which is usually not prepared to support high vehicles loads. Within this context, excessively abrupt shift of bottlenecks indicates areas where the structural transition is excessively sharp, and this must have 
implications, yet to be studied, with respect to the efficiency (in whatever terms) of the transportation system.

Our work leaves open several interesting research avenues. Here we have only considered a fully deterministic routing protocol (shortest paths), while other alternatives would probably render new insights. We can think, for instance, in the extreme opposite situation, i.e. a random-walk dynamics. Additionally, it would probably deserve special attention the inclusion of realistic origin-destination (OD) matrices. Their introduction would allow us to study the extent to which the phenomenology of abrupt transitions is solely a consequence of the network topology. Furthermore, the role of edge weights deserves to be better investigated. In this work we consider these as the Euclidean distances between the endpoints, but it would be interesting to analyze if the congestion location may be manipulated by means of the weights distribution. Still, concerning the reproduction of the aforementioned empirical results, much effort is needed to understand which actually is the effective origin of the transition beyond edge density: discontinuity in nodes distribution, or in connectivity? Our framework permits to construct synthetic networks with nodes and edges properties sampled from the corresponding empirical distributions, thus constituting a simple playground to scrutinize the relationships between structure and function in urban road networks.

\section{Abbreviations}

DT: Delaunay triangulation; MST: Minimum spanning tree.

Acknowledgements

Not applicable.

Authors' contributions

All the authors contributed equally. All authors read and approved the final manuscript.

Funding

A.L., J.B-H. and A.S-R. acknowledge the support of the Spanish MICINN project (Grant PGC2018-096999-A-I00). S.G. acknowledges financial support from Spanish MINECO (Grant PGC2018-094754-B-C21), Generalitat de Catalunya (Grant 2017SGR-896), and Universitat Rovira i Virgili (Grant 2019PFR-URV-B2-41).

Availability of data and materials

The codes used during the current study are available from the corresponding author on reasonable request.

\section{Declarations}

Competing interests

The authors declare that they have no competing interests.

\section{Author details}

${ }^{1}$ Internet Interdisciplinary Institute (IN3), Universitat Oberta de Catalunya, Barcelona, Catalonia, Spain. ${ }^{2}$ Departament d'Enginyeria Informàtica i Matemàtiques, Universitat Rovira i Virgili, Tarragona, Catalonia, Spain. ${ }^{3}$ URPP Social Networks, University of Zurich, Zurich, Switzerland.

Received: 2 March 2021 Accepted: 28 May 2021

Published online: 09 June 2021

\section{References}

Batty M (2012) Building a science of cities. Cities 29:9-16. https://doi.org/10.1016/j.cities.2011.11.008

Chen J, Hu M-B, Li M (2020) Traffic-driven epidemic spreading in multiplex networks. Phys Rev E 101(1):012301

Daganzo CF, Geroliminis N (2008) An analytical approximation for the macroscopic fundamental diagram of urban traffic. Transp Res Part B Methodol 42(9):771-781. https://doi.org/10.1016/j.trb.2008.06.008

Dong J-Q, Huang Z-G, Zhou Z, Huang L, Wu Z-X, Do Y, Wang Y-H (2012) Enhancing transport efficiency by hybrid routing strategy. Europhys Lett (EPL) 99(2):20007 
Echenique P, Gómez-Gardeñes J, Moreno Y (2005) Dynamics of jamming transitions in complex networks. Europhys Lett (EPL) 71(2):325-331. https://doi.org/10.1209/epl/i2005-10080-8

Godfrey J (1969) The mechanism of a road network. Traffic Eng Control 8(8)

Guimerà R, Díaz-Guilera A, Vega-Redondo F, Cabrales A, Arenas A (2002) Optimal network topologies for local search with congestion. Phys Rev Lett 89:248701. https://doi.org/10.1103/PhysRevLett.89.248701

Helbing D (2015) Traffic theory from first principles. Available at SSRN 2960224

Kirkley A, Barbosa H, Barthelemy M, Ghoshal G (2018) From the betweenness centrality in street networks to structural invariants in random planar graphs. Nat Commun 9:2501. https://doi.org/10.1038/s41467-018-04978-z

Lampo A, Borge-Holthoefer J, Gómez S, Solé-Ribalta A (2021) Multiple abrupt phase transitions in urban transport congestion. Phys Rev Res 3:013267. https://doi.org/10.1103/PhysRevResearch.3.013267

Liu Z, Hu M-B, Jiang R, Wang W-X, Wu Q-S (2007) Method to enhance traffic capacity for scale-free networks. Phys Rev E 76(3):037101

Manfredi S, Di Tucci E, Latora V (2018) Mobility and congestion in dynamical multilayer networks with finite storage capacity. Phys Rev Lett 120(6):068301

Masucci AP, Stanilov K, Batty M (2013) Limited urban growth: London's street network dynamics since the 18th century. PLoS ONE 8(8):69469. https://doi.org/10.1371/journal.pone.0069469

Solé-Ribalta A, Arenas A, Gómez S (2019) Effect of shortest path multiplicity on congestion of multiplex networks. New J Phys 21(3):035003

Solé-Ribalta A, Gómez S, Arenas A (2016) Congestion induced by the structure of multiplex networks. Phys Rev Lett 116:108701. https://doi.org/10.1103/PhysRevLett.116.108701

Solé-Ribalta A, Gómez S, Arenas A (2016) A model to identify urban traffic congestion hotspots in complex networks. R Soc Open Sci 3(10):160098

Solé-Ribalta A, Gómez S, Arenas A (2018) Decongestion of urban areas with hotspot pricing. Netw Spatial Econ 18(1):3350. https://doi.org/10.1007/s11067-017-9349-y

Strano E, Cardillo A, lacoviello V, Latora V, Messora R, Porta S, Scellato S (2009) Street centrality and densities of retail and services in Bologna, Italy. Environ Plan 38:450. https://doi.org/10.1068/b34098

Tan F, Wu J, Xia Y, Chi KT (2014) Traffic congestion in interconnected complex networks. Phys Rev E 89(6):062813

Yan G, Zhou T, Hu B, Fu Z-Q, Wang B-H (2006) Efficient routing on complex networks. Phys Rev E 73(4):046108

Zhao L, Lai Y-C, Park K, Ye N (2005) Onset of traffic congestion in complex networks. Phys Rev E 71(2):026125

\section{Publisher's Note}

Springer Nature remains neutral with regard to jurisdictional claims in published maps and institutional affiliations.

\section{Submit your manuscript to a SpringerOpen ${ }^{\circ}$ journal and benefit from:}

- Convenient online submission

Rigorous peer review

Open access: articles freely available online

- High visibility within the field

Retaining the copyright to your article

Submit your next manuscript at $\gg$ springeropen.com 